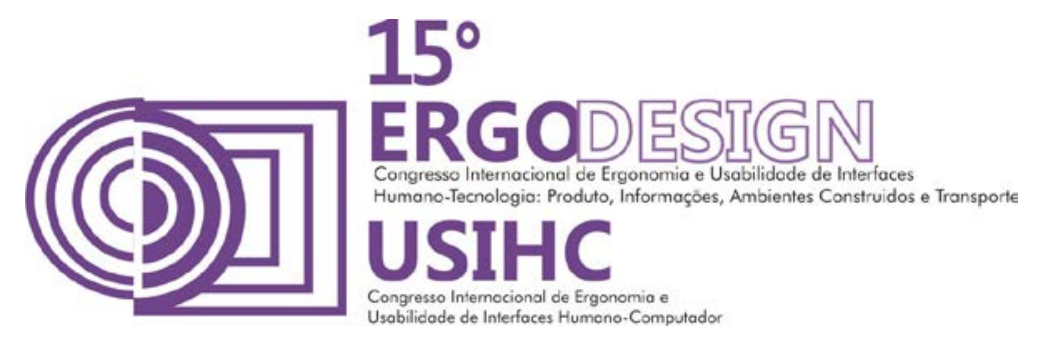

\title{
ROÇADEIRAS COSTAIS MOTORIZADAS: ANÁLISES ESTATÍSTICAS DAS VARIÁVEIS ERGONÔMICAS AVALIADAS
}

\section{BRUSHCUTTERS COSTAL MACHINE: EVALUATED ERGONOMIC STATISTICS VARIABLES ANALYSIS}

\author{
Schutzer, Victor Manieri (1); \\ Santos, João Eduardo Guarnetti dos (2); \\ Paschoarelli, Luis Carlos (3).
}

(1) Universidade Estadual Paulista "Júlio de Mesquita Filho", graduando em Engenharia Mecânica

e-mail:victor schutzer@hotmail.com

(2) Universidade Estadual Paulista "Júlio de Mesquita Filho", Engenheiro Agrícola, Prof. Adjunto Departamento de Engenharia Mecânica UNESP - Bauru

e-mail:guarneti@feb.unesp.br

(3) Universidade Estadual Paulista "Júlio De Mesquita Filho", Livre-Docente em Design

Ergonômico, Docente no Curso de Graduação de Design

e-mail: paschoarelli@faac.unesp.br

\begin{abstract}
RESUMO
Com o aumento da mecanização na agricultura, torna-se cada vez mais frequente e necessária a preocupação com o bem estar dos trabalhadores e, consequentemente, com os aspectos ergonômicos. Para o presente trabalho, foi observada e feita uma análise dos riscos físicos que a vibração e o ruído causam em operadores de roçadeiras costais motorizadas. As análises foram feitas a partir da NR-15 para operações insalubres e a ISO 5349 (2001) - Vibrações mecânicas - medição e avaliação da exposição humana à vibração transmitida à mão.
\end{abstract}

Palavras-chave: Roçadeira Costal, Vibração, Ruído

\section{ABSTRACT}

As the mechanization in agriculture increases, the concerns for the workers' welfare and hence with the ergonomic aspects becomes increasingly common and necessary. This work observed and analyzed the physical risks that cause vibration and noise for operators of motorized coastal brushcutters. Analyses were made on NR - 15 for unhealthy operations and ISO 5349 (2001) - Mechanical vibration measurement and evaluation of human exposure to hand- transmitted vibration.

Keywords: Backpack Brushcutte, Vibration, Noise 


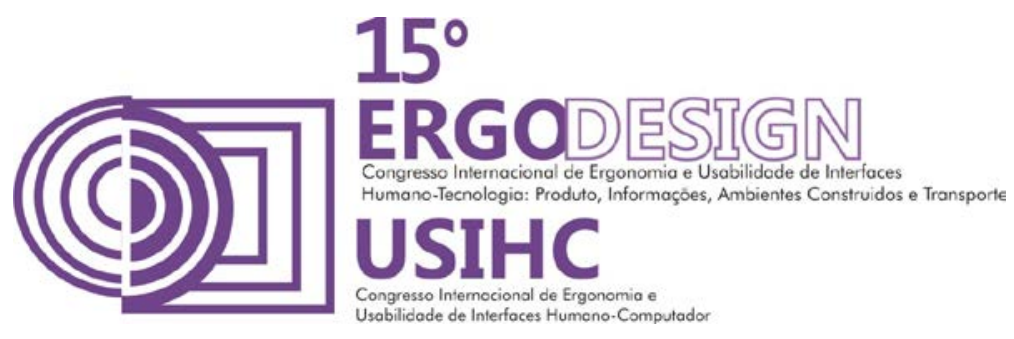

\section{INTRODUÇÃO}

Atualmente tem-se aumentado a preocupação com a saúde, bem-estar e a qualidade de vida dos trabalhadores durante suas atividades laborais. A Ergonomia é umas das ciências que realiza tal estudo. A ergonomia é o estudo da adaptação do trabalho ao homem, em que se observa que a adaptação sempre ocorre do trabalho para o homem, pois é muito mais difícil adaptar o homem ao trabalho (IIDA, 2001). Outra definição é "A Ergonomia é o estudo científico da relação entre o homem, os seus meios, métodos e espaços de trabalho. O seu objetivo é elaborar, mediante a contribuição de diversas disciplinas científicas que a compõem, um corpo de conhecimentos que, dentro de uma perspectiva de aplicação, deve resultar numa melhor adaptação ao homem dos meios tecnológicos e dos ambientes de trabalho e de vida" (IEA, 1989). Novamente, o estudo e a implementação da ergonomia são recentes, ou seja, ainda existem muitas máquinas e equipamentos que são nocivos à saúde do operador. Com isso, o foco do trabalho é avaliação, de formar a medir da forma mais correta possível, verificar irregularidades que ocorram durante a medição e levantar dados para o estudo.

Primeiramente, discutiu-se a importância à exposição ao ruído, que é definido como um som indesejável (IIDA, 2001). Existem algumas formas de controle do som que podem ser: na fonte, na trajetória (medidas no ambiente) e no homem (SALIBA E CORRÊA, 2002). E este controle é de fundamental importância pois, o ruído é um dos itens mais importantes da saúde ocupacional, estando, quando inadequado, relacionado às lesões do aparelho auditivo, à fadiga auditiva e, provavelmente, aos efeitos psicofisiológicos negativos associados ao estresse psíquico (WACHOWICZ, 2007). A perda ou redução da capacidade auditiva pode ocorrer geralmente de maneira temporária, permanecendo por um período logo após uma exposição ao ruído, mas também tende a ocorrer de maneira irreversível quando a exposição ocorre de maneira prolongada, como no passar de anos (GUERRA et al., 2004).

Os primeiros estudos sobre vibração foram motivados por problemas de desbalanceamento de motores e eixos (SOEIRO, 2011). A maioria dos motores de acionamento tem problemas de vibração em razão do desbalanceamento inerente aos motores. Muitos sistemas de engenharia, um ser humano age como parte integral do sistema que resulta em desconforto e perda de eficiência. Na engenharia, os estudos das vibrações são de grande importância e podem ser responsáveis por prejuízos econômicos e financeiros (RAO, 2008).

Posteriormente, verifica-se que a vibração é qualquer movimento que o corpo executa em torno de um ponto fixo. Esse movimento pode ser regular, do tipo senoidal ou irregular, quando não segue nenhum padrão determinado (IIDA, 2001). A vibração é determinada por três variáveis: a frequência, medida em ciclos por segundo ou hertz $(\mathrm{Hz})$; a intensidade do deslocamento (em $\mathrm{cm}$ ou $\mathrm{mm})$ ou aceleração máxima sofrida pelo corpo, medida em $\mathrm{g}(1 \mathrm{~g}=9,81 \mathrm{~m} / \mathrm{s} 2)$. A terceira variável é a direção do movimento, definida por três eixos tri-ortogonais: $x$ (das costas para frente), y (da direita para a esquerda) e o z (dos pés à cabeça) (IIDA, 2001). Já para Kroemer e Grandjean (2005), as vibrações são oscilações da massa em função de um ponto fixo, podem serem produzidas por movimentos periódicos regulares ou irregulares de uma ferramenta, veículo ou outro mecanismo em contato com o corpo humano, deslocando-o da sua posição de repouso.

Mais especificamente para os membros superiores, ferramentas motorizadas e utilizadas durante anos podem ocasionar problemas nas mãos e braços e, a frequência da vibração é tido 




como fator decisivo (KROEMER E GRANDJEAN, 2005). Neste caso podem apresentar a patologia popularmente conhecida como "dedo branco", causando perda da capacidade manipulativa e o tato nas mãos e dedos, dificultando o controle motor (GERGES, 2005). A vibração é algo extremamente prejudicial para o ser humano porém, eliminar a vibração dos ambientes de trabalho é uma proposta quase que impossível, já que todos os equipamentos geram algum tipo de vibração, sendo importante, então, concentrar estudos e esforços para minimizar os riscos e os efeitos nas pessoas (VENDRAME, 2009).

\section{NORMALIZAÇÃO}

\subsection{Vibração}

Em 2001 foi publicada a Norma ISO 5349 (2001), com uma nova metodologia de medição das vibrações em mãos e braços, sem indicar os limites de tolerância para um trabalho seguro.Para ISO 5349 (2001), as medições de vibração são feitas na mão ou próxima do local em que a vibração é transmitida ao corpo como mostra a Figura 1.

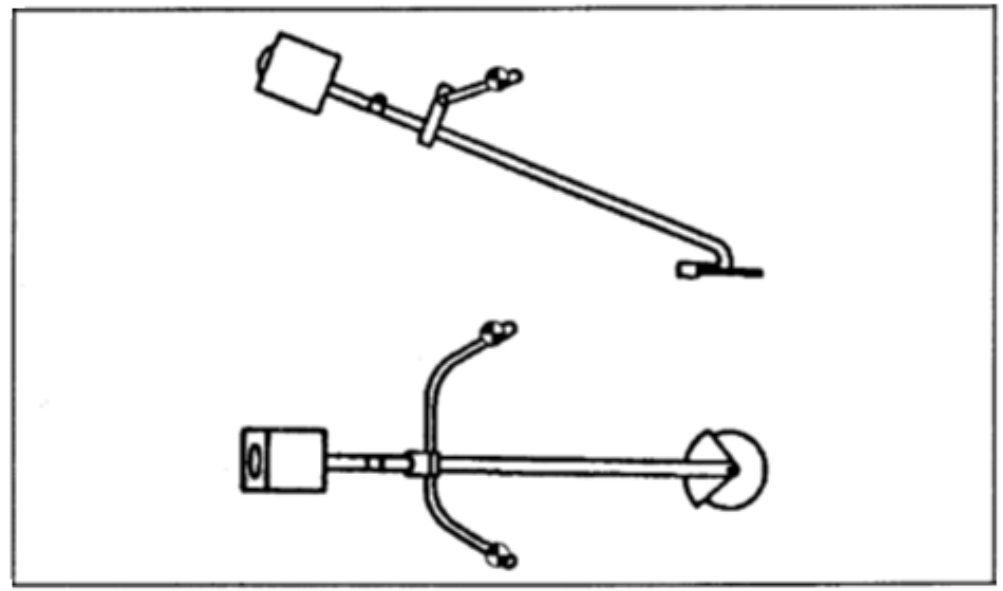

Figura 1. Locais onde se fixa o acelerômetro.

É importante, segundo a ISO 5349 (2001),que se faça medições para todas as ferramentas ou situações para que possa dar uma contribuição significativa para a exposição diária à vibrações. E dessa forma, foi verificada a vibração nas situações em que existiu e não existiu o corte da braquiária, já que o operador deste tipo de equipamento está sujeito a essas duas variações de trabalho

É importante, para a ISO 5349 (2001), que se faça a medição de forma que esta seja representativa para o período em que há o uso da ferramenta e que, o tempo de medição seja de pelo menos de 1 minuto, assim como fez-se no atual trabalho.

Para efeito de cálculos para ISO 5349 (2001), tem-se: 


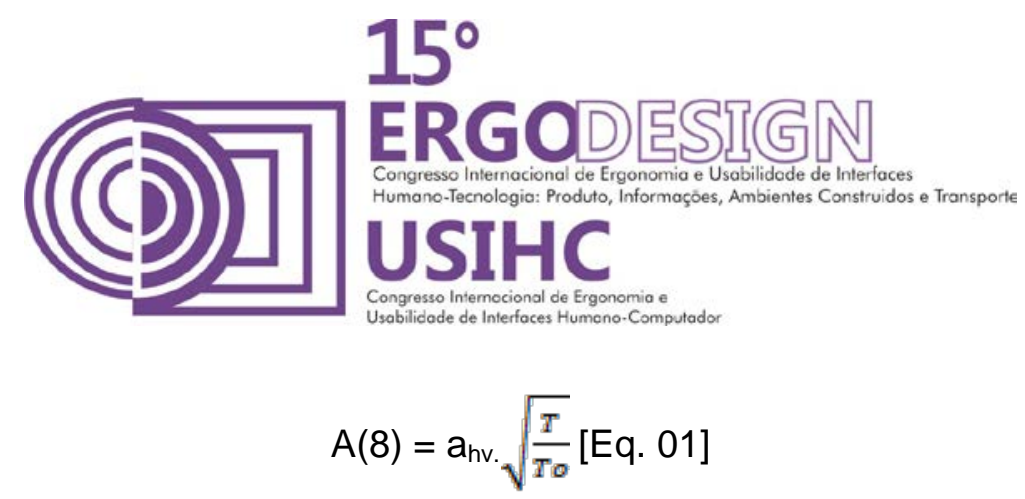

Onde: $\mathrm{A}(8)$ - Projeção de dose para 8 horas;

$a_{\text {hv }}$ valor de aceleraçãoda vibraçãotransmitidaà mão, em metros por segundoao quadrado $(\mathrm{m} / \mathrm{s} 2)$ e RMS;

T - duração total para a exposição da vibração anv; To - duração referente a 8 horas (28800s).

\subsection{Ruído}

Para o ruído tem-se a NR-15 (1978), que fixa uma máxima exposição de $85 \mathrm{~dB}(\mathrm{~A})$ para o trabalho de 8 horas. Conforme aumenta-se o nível de ruído, a máxima exposição diminui gradativamente.

\section{OBJETIVO}

O objetivo do presente trabalho foi mensurar e analisar a vibração que é transmitida das roçadeiras costais motorizadas para a mão do operador quando operam esta máquina durante suas atividades laborais e o ruído que os operadores estão expostos também no decorrer do trabalho. Inconvenientes, estes, que são inerentes a este tipo de trabalho realizado com essas máquinas. Para as análises dos dados, foram utilizados para o ruído a norma NR-15 (1978) e a norma ISO 5349 (2001) para a vibração e os resultados foram avaliados segundo a Diretiva 2002/44/EC (2002).

\section{MATERIAIS E MÉTODOS}

\subsection{Materiais}

Utilizou-se para aquisição de dados da vibração um equipamento modelo HVM 100, marca Larson Davis, que foi fixado na empunhadura do equipamento, muito próximo da mão do operador conforme recomendações das normas vigentes, em condições de operação, não interferindo no trabalho normal do operador, sendo que durante as avaliações realizadas os operadores estavam utilizando os equipamentos de proteção recomendados para a realização das operações. O equipamento utilizado realiza leituras simultâneas triaxiais, possibilitando configurações independentes para cada eixo de acordo com o manual do usuário e bibliografias correlatadas e os resultados foram analisados com o software Blase. Conforme pode ser observado na Figura 2. 

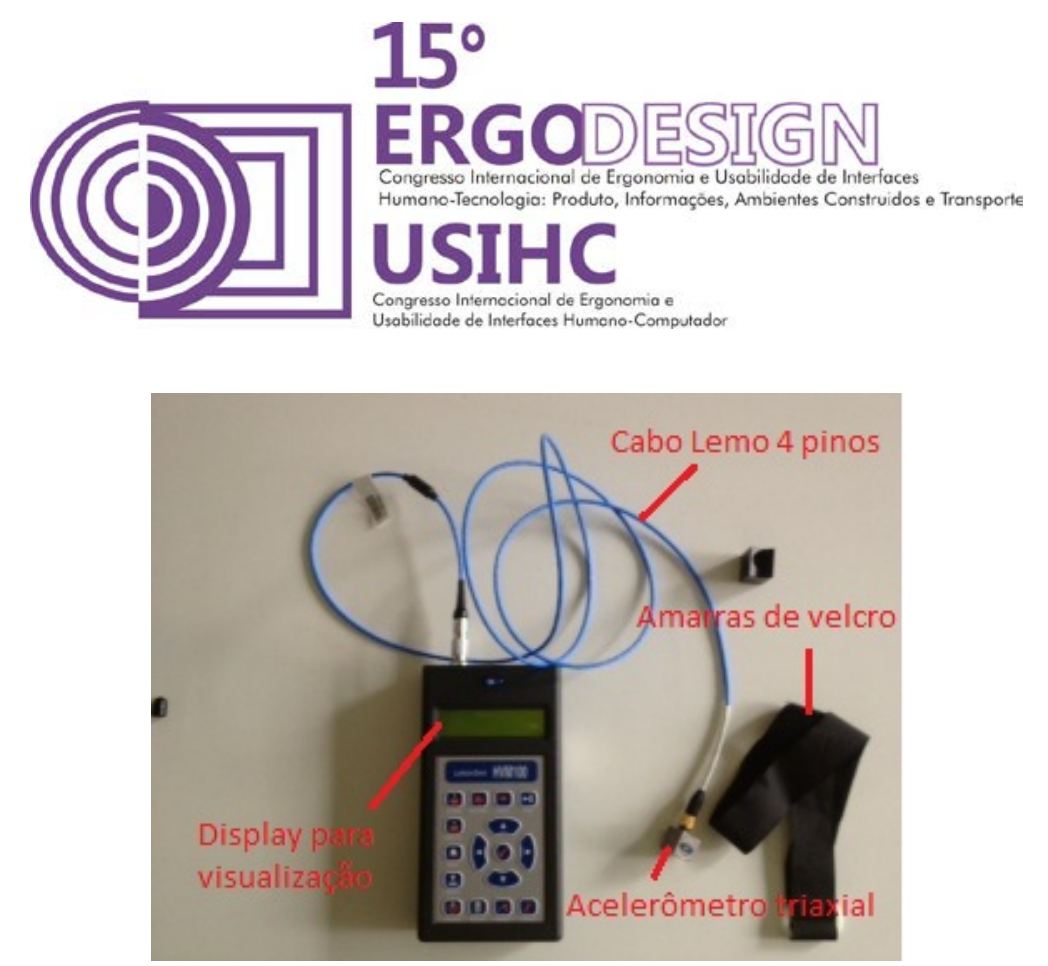

Figura 2. Acelerômetro HVM-100 e seus acessórios.

Foram utilizadas 6 (seis) roçadeiras transversais motorizadas da marca STIHL FS 160 e 1 (uma) roçadeira costal motorizada da marca MAKITA RBC412U. O acelerômetro foi instalado conforme a Norma ISO 5349 (2001).

Para medição do ruído utilizou-se um decibelímetro da marca Instrutherm, DEC-300, equipamento este pertencente ao Departamento de Engenharia Mecânica da FE/UNESP Campus de Bauru, como mostra a Figura 3.

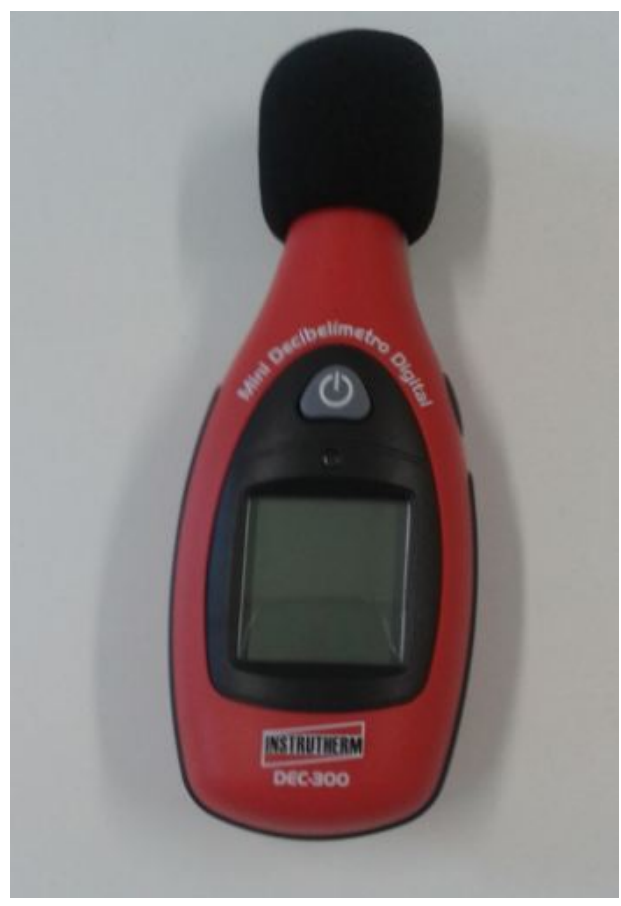

Figura 3. Decibelímetro DEC-300. 


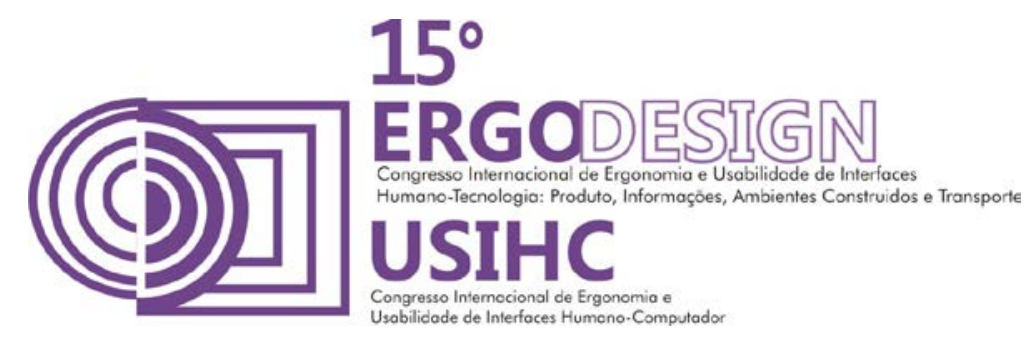

\subsection{Métodos}

Os procedimentos desta pesquisa foram aprovados pelo comitê de Ética da Faculdade de Ciências da Unesp - campus de Bauru, processo número 31410214.8.0000.5398, parecer número 681.964 e um Termo de Consentimento Livre e Esclarecido foi utilizado.

Para avaliação dos resultados, utilizou-se para vibração a norma ISO 5349 (Guia para medição e avaliação da exposição humana à vibração transmitida à mão, (2001) e para o ruído a NR 15 (Atividades e Operações Insalubres, 1978). No presente trabalho, utilizou-se sete roçadeiras sendo avaliado os parâmetros de ruído e vibração utilizando rotação máxima de acordo com o manual do fabricante.

Após a aplicação da Equação 1, realizou-se a análise dos resultados obtidos considerando-se os valores permissíveis da vibração segundo a Diretiva 2002/44/EC (2002) que estipula os níveis de ação e limites de exposição para vibrações de corpo inteiro e de mãos e braços, de acordo com a Tabela 1.

Tabela 1. Níveis de Ação e Limite de exposição.

\begin{tabular}{|c|c|c|}
\hline & Nível de Ação - A(8) & Limite de exposição - A(8) \\
\hline Mãos e braços & $2,5 \mathrm{~m} / \mathrm{s}^{2}$ & $5,0 \mathrm{~m} / \mathrm{s}^{2}$ \\
\hline
\end{tabular}

Foi considerado durante o tempo de avaliação da vibração interrupções (pausas) seja para a troca do nylon (elemento cortante) da roçadeira, seja para reposicionar a máquina para o local adequado e até mesmo para o abastecimento de combustível e operações de manutenção. Entretanto, pela dificuldade de se mensurar o tempo correto da realização do corte, apenas foi descontado o tempo de intervalo de 20 minutos em cada turno de trabalho do operador, totalizando 440 minutos de exposição à vibração.

A avaliação da vibração foi realizada na superfície da mão onde a energia transfere-se para o corpo. Para a realização dos testes a nível de campo, utilizou-se luvas para diminuir a vibração sentida pela mão do operador.

Para a análise da vibração transmitida pela roçadeira costal motorizada, realizou-se duas medições: primeiramente avaliou-se a vibração que a roçadeira transmitia à mão do operador sem a realização do corte e com uma rotação de trabalho constante. A seguir, realizou-se o corte do capim braquiária em condições normais de trabalho, com uma rotação de trabalho de 12000 rpm (alta rotação).

Para avaliar o nível de ruído, realizou-se a medição em quatro 4 locais distintos: à frente, atrás, do lado esquerdo e do lado direito do operador. Esses pontos foram escolhidos para poder representar o ruído que está chegando aos ouvidos do operador (lado esquerdo e lado direito), para medir o local mais próximo de onde está sendo gerado o ruído pelo motor (atrás) e para representar o som que está se propagando na região próxima do operador (à frente). Esses 


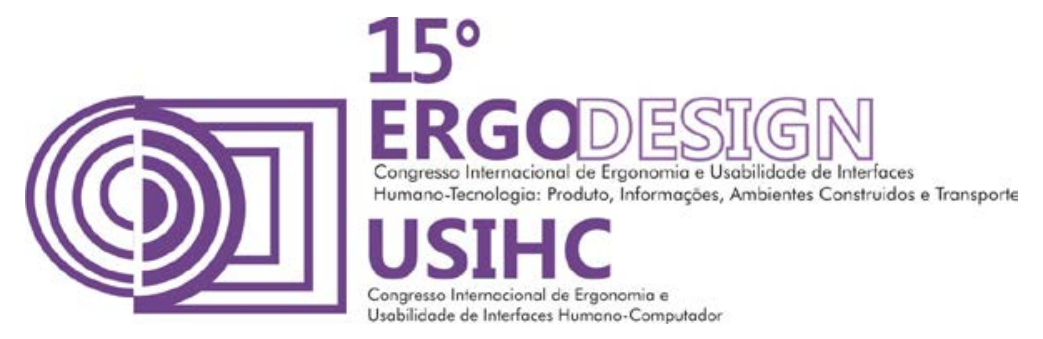

pontos de medição estão de acordo com a NBR 9999 (1987), que trata da medição do nível de ruído, no posto de operação, de tratores e máquinas agrícolas.

Posicionou-se o decibelímetro a uma distância em torno de $15 \mathrm{~cm}$ do operador na medição dos 4 (quatro) pontos distintos, e com um tempo de medição suficiente para que estabilizasse a operação medida. Para o desenvolvimento do presente trabalho, preocupou-se em representar as condições reais que os operadores deste tipo de máquina enfrentam no dia-a-dia de trabalho. Para minimizar erros de medição, utilizou-se um único operador para operar as roçadeiras avaliadas. Os levantamentos de dados a nível de campo foram realizados em um único dia com $100 \%$ de umidade do ar e temperatura em torno de $16,4^{\circ} \mathrm{C}$, dados estes obtidos no site do IPMET (Instituto de Pesquisas Meteorológicas em 16/09/2013 dia da realização dos ensaios). Foi considerada a massa verde no período exato em que, normalmente, este tipo de vegetação é cortado pelos trabalhadores. O HVM-100 foi calibrado de acordo com 0 especificado pelo fabricante considerando-se as normas específicas.

\section{RESULTADOS E DISCUSSÃO}

\subsection{Vibração}

Para avaliação da vibração, foi gerada a Tabela 2, de acordo com a ISO 5349 (2001) e a Diretiva 2002/44/EC (2002) na qual pode-se classificar cada máquina e situação (com e sem corte), se estão abaixo ou acima do limite de exposição.

Tabela 2. Avaliação da vibração.

\begin{tabular}{|c|c|c|c|c|}
\hline Máquina & Situação & $\begin{array}{c}\text { Aceleração } \\
\left(\mathbf{m} / \mathbf{s}^{\mathbf{2}} \mathbf{)}\right.\end{array}$ & $\begin{array}{c}\mathbf{A}(\mathbf{8}) \\
\left(\mathbf{m} / \mathbf{s}^{\mathbf{2}}\right)\end{array}$ & Classificação \\
\hline Stihl FS160 1 & Sem Corte & 4.58 & 4.39 & Abaixo do limite de exposição \\
\hline Stihl FS160 1 & Com Corte & 3.64 & 3.49 & Abaixo do limite de exposição \\
\hline Stihl FS160 2 & Sem Corte & 6.16 & 5.90 & Acima do limite de exposição \\
\hline Stihl FS160 2 & Com Corte & 6.03 & 5.77 & Acima do limite de exposição \\
\hline Stihl FS160 3 & Sem Corte & 3.47 & 3.32 & Abaixo do limite de exposição \\
\hline Stihl FS160 3 & Com Corte & 3.41 & 3.27 & Abaixo do limite de exposição \\
\hline Stihl FS160 4 & Sem Corte & 3.75 & 3.59 & Abaixo do limite de exposição \\
\hline Stihl FS160 4 & Com Corte & 3.71 & 3.55 & Abaixo do limite de exposição \\
\hline Stihl FS160 5 & Sem Corte & 5.37 & 5.14 & Acima do limite de exposição \\
\hline
\end{tabular}




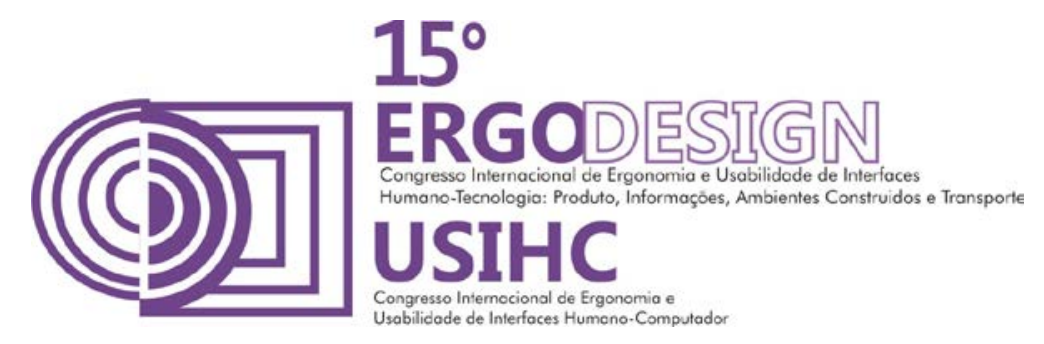

\begin{tabular}{|c|c|c|c|c|}
\hline Stihl FS160 5 & Com Corte & 7.17 & 6.87 & Acima do limite de exposição \\
\hline Stihl FS160 6 & Sem Corte & 2.27 & 2.17 & Abaixo do limite de exposição \\
\hline Stihl FS160 6 & Com Corte & 2.95 & 2.82 & Abaixo do limite de exposição \\
\hline Makita RBC 412U 7 & Sem Corte & 14.50 & 13.88 & Acima do limite de exposição \\
\hline Makita RBC 412U 7 & Com Corte & 6.78 & 6.49 & Acima do limite de exposição \\
\hline
\end{tabular}

Com os resultados acima, tanto para a realização do corte ou sem o corte, temos que para todos as medições realizadas, exceto na vibração para o trabalho sem corte da Máquina 6 (única medida que está abaixo de $2.5 \mathrm{~m} / \mathrm{s}^{2}$ ), será necessário que o operador tome medidas para que diminua a vibração que é captada por sua mão. Estas medidas são de fundamental importância para a saúde do operador, para que este não venha a ter problemas de saúde típicos de quem está exposto às altas vibrações nas mãos e braços.

Para um melhor entendimento, realizou-se a análise estatística dos testes, como observa-se na Tabela 3:

Tabela 3. Análise estatística da vibração.

\begin{tabular}{|c|c|c|}
\hline \multirow{2}{*}{ Máquina } & \multicolumn{2}{|c|}{ Situação } \\
\cline { 2 - 3 } & Sem Corte & Com Corte \\
\hline 1 & 4,58 & 3,64 \\
\hline 2 & 6,16 & 6,03 \\
\hline 3 & 3,47 & 3,41 \\
\hline 4 & 3,75 & 3,71 \\
\hline 5 & 5,37 & 7,17 \\
\hline 6 & 2,27 & 2,95 \\
\hline 7 & 14,50 & 6,78 \\
\hline Análise Estatística & \multicolumn{2}{|c}{} \\
\hline Média & 5,73 & 4,81 \\
\hline Mediana & 4,58 & 4,71 \\
\hline Amplitude & 12,23 & \\
\hline Coeficiente de Variação & \multicolumn{2}{|c}{5,27} \\
\hline Média Geral & \multicolumn{2}{|c|}{} \\
\hline
\end{tabular}




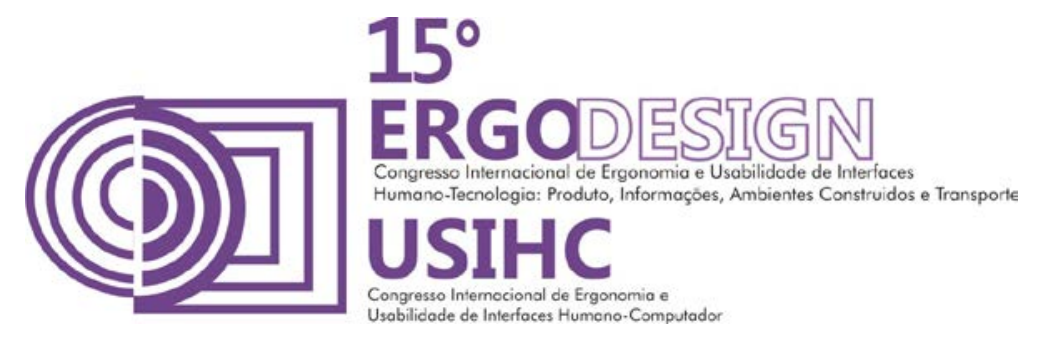

Percebe-se um Coeficiente de Variação de 41,83\%. Esse alto Coeficiente de Variação se deve ao número elevado de fatores que interferem no bom funcionamento da máquina.

\subsection{Ruído}

O ruído foi medido para todas as máquinas, sendo as posições: frente, atrás, lado esquerdo e lado direito. Os resultados obtidos podem ser vistos na Figura 4 em que se verifica o ruído de acordo com cada posição medida para a respectiva máquina. Foram considerados apenas os valores máximos atingidos durante a medição. Através da figura abaixo, pode-se visualizar a variação da intensidade do ruído para cada ponto de medição e para a máquina operando ou não.

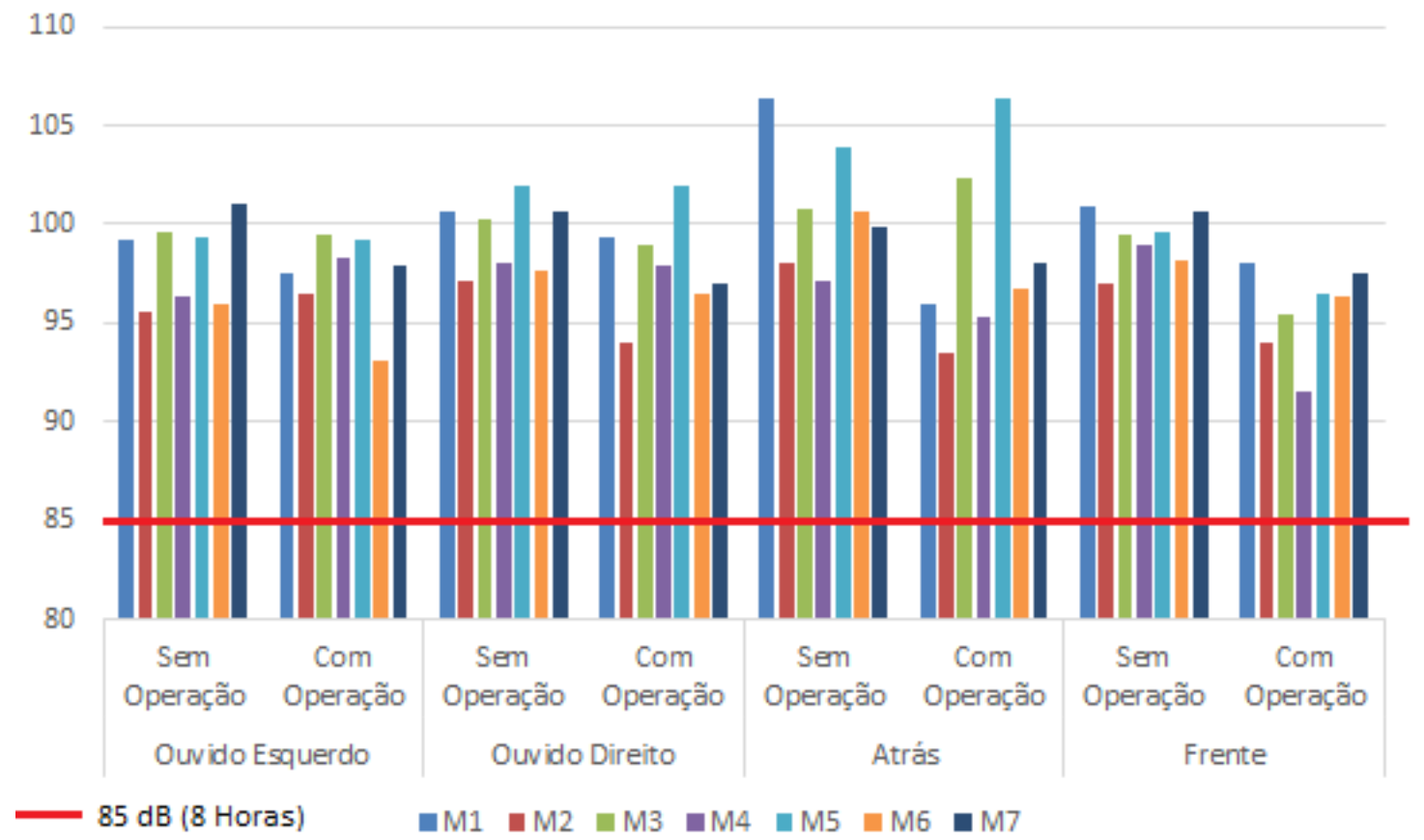

Figura 4. Pontos de medição e ruído captado.

Para uma melhor visualização da situação em que o operador utiliza protetor auricular do tipo Concha, estabeleceu-se a Figura 5 com redução de 20 db (SUCEN, 2008). A NRRsf (Nível de Redução de Ruído "subject fit") foi subtraída de todas as medições e avaliada de acordo com a NR-15 (1978). 

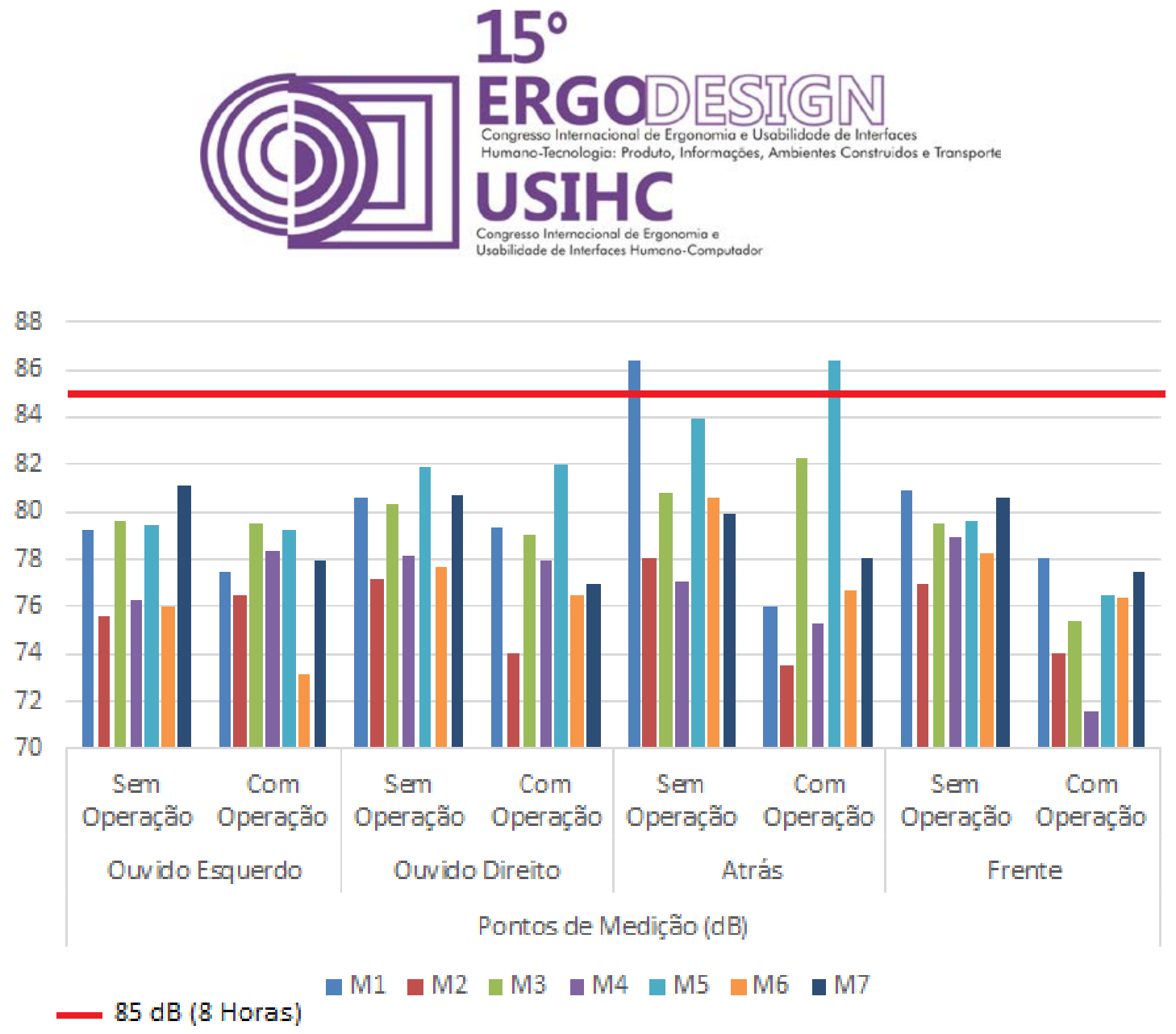

Figura 5. Pontos de medição e ruído captado com operador utilizando protetor auricular do tipo Concha.

Como dito anteriormente, foi considerado apenas os valores máximos do ruído nas quatro posições distintas. Com isso, e com a Figura 5 percebe-se que, com o operador utilizando protetor auricular do tipo Concha, quase todo o ruído captado pelo ouvido do mesmo está abaixo de 85 dB. Portanto, segundo a NR-15 (1978), o operador pode trabalhar durante 8 horas sem qualquer dano à saúde. Para uma melhor visualização dos resultados, foi gerada a Tabela 4 com a análise estatística do ruído.

Tabela 4. Análise estatística da ruído.

\begin{tabular}{|c|c|c|c|c|c|c|c|c|}
\cline { 2 - 9 } \multicolumn{1}{c|}{} & \multicolumn{9}{c|}{ Pontos de Medição (dB) } \\
\hline \multirow{2}{*}{ Máquina } & \multicolumn{2}{|c}{ O.E. } & \multicolumn{2}{c|}{ O.D. } & \multicolumn{2}{c|}{ Atrás } & \multicolumn{2}{c|}{ Frente } \\
\cline { 2 - 9 } & S.O. & C.O & S.O. & C.O. & S.O. & C.O. & S.O. & C.O. \\
\hline 1 & 99,20 & 97,50 & 100,60 & 99,30 & 106,40 & 96,00 & 100,90 & 98,00 \\
\hline 2 & 95,60 & 96,50 & 97,20 & 94,00 & 98,00 & 93,50 & 97,00 & 94,00 \\
\hline 3 & 99,60 & 99,50 & 100,30 & 99,00 & 100,80 & 102,30 & 99,50 & 95,40 \\
\hline 4 & 96,30 & 98,30 & 98,10 & 97,90 & 97,10 & 95,30 & 98,90 & 91,60 \\
\hline 5 & 99,40 & 99,20 & 101,90 & 102,00 & 103,90 & 106,40 & 99,60 & 96,50 \\
\hline 6 & 96,00 & 93,10 & 97,70 & 96,50 & 100,60 & 96,70 & 98,20 & 96,40 \\
\hline 7 & 101,10 & 97,90 & 100,70 & 97,00 & 99,90 & 98,00 & 100,60 & 97,50 \\
\hline
\end{tabular}




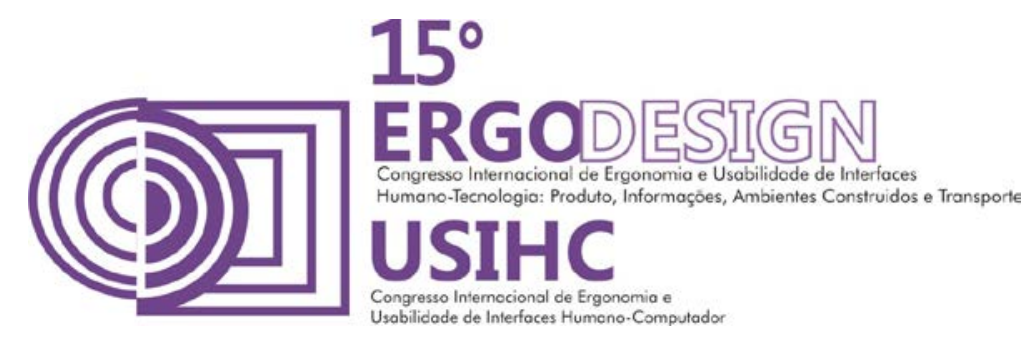

\begin{tabular}{|c|c|c|c|c|c|c|c|c|}
\hline Análise & & & & & & & & \\
\hline Média & 98,17 & 97,43 & 99,50 & 97,96 & 100,96 & 98,31 & 99,24 & 95,63 \\
\hline Mediana & 99,20 & 97,90 & 100,30 & 97,90 & 100,60 & 96,70 & 99,50 & 96,40 \\
\hline Amplitude & 5,50 & 6,40 & 4,70 & 8,00 & 9,30 & 12,90 & 3,90 & 6,40 \\
\hline $\mathrm{CV}$ & \multicolumn{2}{|c|}{$1,40 \%$} & \multicolumn{2}{|c|}{$1,02 \%$} & \multicolumn{2}{|c|}{$2,01 \%$} & \multicolumn{2}{|c|}{$1,28 \%$} \\
\hline Média Geral & \multicolumn{2}{|c|}{97,80} & \multicolumn{2}{|c|}{98,73} & \multicolumn{2}{|c|}{99,21} & \multicolumn{2}{|c|}{97,44} \\
\hline
\end{tabular}

Percebe-se através da Tabela 4 que houve um pequeno Coeficiente de Variação nos testes. Ao contrário da vibração, o ruído apresentou-se de forma mais ou menos constante no decorrer dos testes.

\section{CONCLUSÃO}

É importante que o operador esteja atento à ISO 5349 (2001) que estabelece os níveis de ação e limite exposição de acordo com a vibração que é transmitida à mão do operador.

O ruído medido apresentou altos índices de riscos para o operador se não tomar os devidos cuidados. O tempo máximo de exposição ao ruído que é estipulado pela NR-15 (1978) deve ser corretamente obedecido para que não haja maiores problemas com a saúde do operador.

Para que possa maximizar o tempo de exposição do trabalhador ao ruído e, consequentemente, diminuir os riscos à saúde do operador, é de fundamental importância que use o protetor auricular para minimizar a intensidade do ruído captado pelo ouvido do operador.

Não menos importante, são os trabalhadores que estão em volta do operador da máquina fazendo seus trabalhos. Estes necessitam, também, do uso de todos os EPIs necessários para a sua proteção.

Pode-se concluir que, para ambas as variáveis do processo de corte (vibração e ruído), necessita-se o uso correto de EPIs. É de grande importância que a roçadeira possua uma haste emborrachada para minimizar a transmissão de vibração ao operador e, além disso, que o operador utilize luvas. Em relação ao ruído, o operador deve estar usando, em tempo integral, o protetor auricular para que minimize o ruído captado pelo ouvido do trabalhador.

\section{REFERÊNCIAS BIBLIOGRÁFICAS}

ABNT - ASSOCIAÇÃO BRASILEIRA DE NORMAS TÉCNICAS - NORMA NBR 9999 - Medição do Nível de Ruído no Posto de Operação de Tratores e Máquinas Agrícolas. 1987.

BRASIL. Ministério do Trabalho e Emprego. Portaria 3.214 - Normas Regulamentadoras, 1978.

DIRETIVA EUROPEIA 2002/44/EC - Parlamento Europeu e Conselho da União Europeia de 25/06/2002. Jornal Oficial da Comunidade Europeia de 06/07/2002, L 177. Pag. 13-19, 2002.

FERNANDES, J. C.Acústica e ruídos. - Apostila do Departamento de Engenharia Mecânica da UNESP - Campus de Bauru. UNESP, 2013, disponível em http://wwwp.feb.unesp.br/jcandido

FERNANDES, J.C. e FERNANDES, M.R.M. Guia Para Medição e Avaliação da Exposição Humana à 


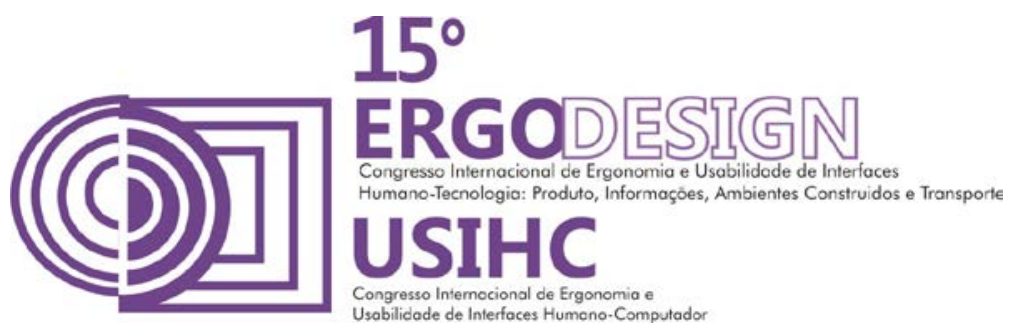

Vibração Transmitida à Mão. Tradução da Norma ISO 5349/1979. Departamento de Engenharia Mecânica da UNESP - Campus de Bauru. UNESP, 2009. Disponível em: <http://wwwp.feb.unesp.br/jcandido $>$

GERGES, S. N.Y.Ruído: Fundamentos e Controle, $1^{\text {a }}$ ed., Florianópolis, 2005

GUERRA, M.R., LOURENÇO, P.M.C., TEIREIRA, M.T.B., ALVES, M.J.M. Prevalência de Perda Auditiva Induzida por Ruído em Empresa Metalúrgica, 2004.

IIDA, I. Ergonomia: Projeto e Produção. 2. Ed. São Paulo: Editora Edgard, Blucher, 2001.

INTERNATIONAL ERGONOMICS ASSOCIATION. Conceito Adotado no Congresso Internacional de Ergonomia, 1989.

ISO - INTERNATIONAL ORGANIZATION FOR STANDARDZATION (ISO 5349) - Mechanical Vibration Measurement and Evaluation of Human Exposure to Hand Transmitted Vibration. Part 1: General Requirements. Part 2: Practical Guidance for Measurement at the Workplace. Genebra, 2001.

KROEMER, K. H. E., GRANDJEAN, E., Manual de Ergonomia: Adaptando o trabalho ao homem, $5^{\circ}$ edição Artmed Editora, Porto Alegre, 2005.

MAKITA. Roçadeiras à Gasolina. Disponível em: <http://www.makita.com.br>

NR 15 - Norma Regulamentadora 15 - Atividades e Operações Insalubres, 1978.

RAO, S. Vibrações Mecânicas. São Paulo: Pearson, 423p.

SALIBA, T. M.; CORRÊA, M. A. C. Insalubridade e Periculosidade - Aspectos Técnicos e Práticos. 6. Ed. São Paulo, Editora LTr, 2002.

SOEIRO, N. S. Vibração e o Corpo Humano: uma avaliação ocupacional. In: $1^{\circ}$ Workshop de vibração e acústica, 2011.

STIHL. Roçadeira Para O Mercado De Jardinagem. Disponível em: <http://www.stihl.com.br>

SUCEN - Superintendência de Controle de Endemias - Avaliação da exposição a ruído e medidas de controle, 2008.

VENDRAME, A. C. Segurança do Trabalho, Saúde e Meio Ambiente, 2005. Disponível em: $<$ http://www.vendrame.com.br/artigos.htm>

VENDRAME, A., C. Vibração Operacional. Revista Proteção. 2009

WACHOWICZ, M. C. Segurança, saúde \& ergonomia. Curitiba, Editora IBPEX, 2007.

\section{AGRADECIMENTOS}

Agradece-se ao CNPq - Conselho nacional de Desenvolvimento Científico e Tecnológico pela concessão da Bolsa de Iniciação Científica processo n 470869/2012-7. 